\title{
New Fluorescent-Histochemical and Immuno-Histochemical Aspects of Secondary Cataract Pathogenesis in Humans
}

\author{
Nadezhda Vitalievna Korsakova \\ Medicine Department, Chuvash State University, Cheboksary, Chuvash Republic, Russia
}

Email address:

korsnv@rambler.ru,korsnv@mail.ru

To cite this article:

Nadezhda Vitalievna Korsakova. New Fluorescent-Histochemical and Immuno-Histochemical Aspects of Secondary Cataract Pathogenesis in Humans. International Journal of Photochemistry and Photobiology. Vol. 5, No. 5, 2017, pp. 80-87. doi: 10.11648/j.iji.20170505.11

Received: June 21, 2017; Accepted: August 7, 2017; Published: November 28, 2017

\begin{abstract}
Secondary cataract is the most common postoperative complication, which causes repeated significant reduced visual acuity, even after a successful operation on the lens. Due to significant clinical heterogeneity of secondary cataract of a man a comparative morphological and functional study in the formation of its fibrous and proliferative forms is especially important for identification of pathogenesis foundations of this disease. The used morphological, histochemical and clinical techniques in the conducted study have provided some evidence of the important role of trophic nervous influences on the type formation of secondary cataract in humans. It is important to note that the revealed similarities in changes of bioamine status, immunoreactivity of the epithelium and fibers of the human crystalline lens in different types of age-related cataract (cortical and nuclear) and its postoperative complications (proliferative and fibrous types of secondary cataract, respectively) can serve as an important proof of their common pathogenesis and, consequently, the type of age-related cataract can be offered as an accessible marker for predicting the risk of postoperative secondary cataract of certain type and its early prevention. The results allow for the first time to put forward a new concept of the secondary cataract pathogenesis. The presented in this chapter concept emphasizes the importance of the fundamental problems of medicine - maintaining the stability of tissue differentiation of a living organism in a variety of physiological and pathological conditions, under the influence of neurotrophic factors.
\end{abstract}

Keywords: Secondary Cataract, Age-Related Cataract, Pathogenesis, Autonomic Nervous System, Neurotransmitters, Phenotype

\section{Introduction}

Commonly there is a significant increase in the cataract incidence, which was recognized as a major (47\%) reason of a visual acuity reduction in the world, and is now regarded as a medical and social problem of national importance. At the age over 60 its initial symptoms were reported in $64-68 \%$ of patients. At the age over 80 practically all people suffer from cataracts. In the world there are about 17 million blind people due to cataracts $[1,2]$.

Operative therapy of cataract is still the main treatment. Advances in cataract surgery are due to constantly improving operative technique, a minimum number of complications. The fundamental issues of causes and mechanisms of cataract formation are remained without proper attention. This trend is exacerbated by the lack of effective conservative methods of cataract prevention and its treatment, a flagrant necessity of which exists in different countries of the world among the elderly and immobile patients with polypathology. For example, in some developing countries only $10 \%$ of those in need are operated.

Unfortunately, up to date the constantly improving operative technique is not deprived of a number of postoperative complications involving a repeated, significant visual acuity reduction (secondary cataracts, cystic retinal dystrophy, and others). Secondary cataract - the most common postoperative complication, which causes a repeated, significant visual acuity reduction, even after a successful operation on the lens. The incidence of secondary cataract ranges from 3 to $87 \%$ [3]. There is even an opinion that if approaches to diagnosis, prevention and treatment of secondary cataract are not improved, it will become the second leading cause of blindness in the world after agerelated cataract. The cause of these complications can not be 
eliminated surgically, as the existing age-related neurodystrophic process (age-related cataract), having at its base the nervous tissue trophism disturbances of constituent tissue organ $[4,5]$, causes its cells not for reparative but pathological regeneration, one manifestation of which is the phenotype change of these cells. It is proved that the lens immunoreactivity is not the same cells normally and in affection with different types of cataract $[6,7,8,9]$, which touches on the fundamental problem of tissue differentiation stability maintaining of a living organism in various physiological and pathological conditions.

The lens regeneration adequacy in different animal species varies greatly - from the small defects restoration to the full recovery of the lost lens. In a human the adequate lens regeneration does not occur, example of this is secondary cataract, which occurs in $22.5 \%$ of patients after successful surgical treatment of age-related cataract. There are the following types of secondary cataract: 1 - the fibrous type or fibrosis of the posterior lens capsule; 2 - the proliferative type or secondary cataract with Adamyuk-Elschnig pearls formation (syn. Elshnig pearls - these are spherical cell conglomerates on the internal surface of the lens capsule, arising from excessive regeneration of the lens epithelial cells remaining after surgical treatment of cataract, which take on an elongated, spherical shape or with appendages and the ability to proliferate and migrate); 3 - the lens capsule thickening [3]. It is important to note that in the postoperative period in patients with age-related cortical cataract the proliferative type of secondary cataract (with AdamyukElschnig pearls formation) is significantly more frequently formed, in patients with age-related nuclear cataracts - the fibrous type (the posterior lens capsule fibrosis) [10, 11]. Consequently, the search for solutions to the problems of the primary age-related and secondary postoperative cataractogenesis is connected with the necessity of lens regeneration modern concepts expansion in the age aspect.

The humoral system influence on the function regulation of the lens cell is important and confirmed by expression of the named cells of the large number of receptors, such as $\mathrm{H}_{1}$ gistamino-, $\quad \mathrm{M}_{1}-, \quad \mathrm{M}_{3}$-choline, $\mathrm{P}_{2} \mathrm{U}-, \mathrm{P}_{2} \mathrm{Y}_{2}$-purine, $\alpha_{1}$ adrenergic receptors, mineralocorticoid, glucocorticoid receptors to sex hormones (estrogen, progesterone, androgen) signaling receptors of the epidermal growth factor and many others $[12,13,14,15]$.

It is known that synthesis of not endocrine epithelial cells of biogenic amines and cell expression of the same receptors to the corresponding biologically active substances testifies to the presence of auto- and paracrine action mechanisms of the latter. An important feature of cells synthesizing biogenic amines (histamine, catecholamines, serotonin, and others), is their high plasticity, including phenotype, manifested in changing over specific syntheses at the functional organism state changing. The little studied aspect of humoral regulation of the lens cells functioning is its bioamines supply. Studying their possible role is especially true, when one considers the already described role of biogenic amines such as histamine in regulation of calcium ions transport across cell membranes, excess of which in the lens cells, as is known, is able to initiate the cortical type development of cataract $[16,17]$. Despite the large number of studies on the problems of pathogenesis and treatment of secondary cataract, the possible role of changes in the status of bioamines lens cell is remained largely unexplored. Therefore, the study of human lens cells in normal conditions and in different types formation of secondary cataract for the biogenic amines content (histamine, catecholamines, serotonin) and immunohistochemical study of their chemical state to monoclonal antibodies to the basic types of body tissues (neuron specific enolase, vimentin, a protein S-100, $\alpha$ smooth muscle actin and epithelial membrane antigen) is an actual problem of modern medicine.

Thus, taking into account the clinical features of different types of secondary cataract, the active participation of neurotransmitter biogenic amines in the lens cells functioning and stability maintenance of their tissue differentiation, the study of bioamines, immuno-histochemical profile of the lens cells of patients in the different types formation of secondary cataract should be considered as specific practical problems of developing pathogenetic methods of its preclinical diagnosis, prevention and therapy.

\section{Objective}

To put forward a new concept of the secondary cataracts pathogenesis in humans and to propose a new method of preclinical prediction.

\section{Material and Methods}

The observations were carried out on 396 lenses of 198 patients of both sexes aged 60-70 years. The research applied methods focused on the study of morphological and clinical features of fibrous and proliferative types formation of secondary cataract in humans.

The material of the capsule, the epithelium, and the substance of lens with cataract, obtained in the planned surgical treatment of age-related cataract of cortical and nuclear types in called patients was studied. The control was 15 lenses, obtained by the eyeballs enucleation in young men corpses aged 20-30 years within 12 hours of death in an accident. The enucleation was carried out with the purpose of the planned donor cornea transplantation.

The continuous statistical clinical study of patients successfully operated for age-related cataract and applying to the hospital within one year after surgery with signs of secondary lens opacity was carried out during the ophthalmologic examination and retrospective analysis of outpatient cards' data of called patients was conducted.

The studied patients were divided into two groups: group A - patients operated on for the cortical type of age-related cataract ( 78 people); group B - patients operated on for the nuclear type of age-related cataract (120 people). These groups of patients were studied with the biomicroscopy method of the anterior eye to identify the type of age-related and secondary cataract using a slit lamp SL-3G-06.

The compulsory condition for this material inclusion in the 
study was the secondary cataract development in a patient for one year after the successfully performed surgery for agerelated cataract. The standard course of operations, provided in the patient signed informed consent, was not impaired. The material for morphological studies was provided under the authority of the Ethics Committee of the Institute of Postgraduate Medical Department of Health and Social Development of the Chuvash Republic (Protocol № 1 of 01.04.2011).

The cryostat sections and paraffin sagittal lens of 15 microns in thickness were treated with the following methods:

1). The hematoxylin-eosin staining was used as general histological staining of the lens.

2 ). In order to identify the histaminocontaining structures the fresh cryostat sections of the lens were treated with the fluorescent-histochemical method of Cross, Evan, Rost [18].

3). The fluorescent-histochemical method of Falk-Hillarp $[19,20]$ for the selective lens structures detection, containing catecholamines and serotonin.

4). The quantitative concentrations of biogenic amines in the various lens structures were determined using cytospectrofluorometry.

5). The complex of immuno-histochemical reactions with monoclonal antibodies to: neural-specific enolase (NSE), protein S-100 (S-100), vimentin (Vim), epithelial membrane antigen (EMA) and $\alpha$-smooth muscle actin ( $\alpha$-SMA). The obtained material during the operation was fixed in $10 \%$ neutral formaldehyde solution. The material was dehydrated in ethanol of gradually increasing concentration and embedded in paraffin. The sections of the lens and capsule with 15 microns in thickness were obtained on the paraffin rotator microtome MPS-2 (JSC KZ 'Tochmedpribor', Ukraine). After deparaffinization and rehydration in ethanol of descending concentration the sections were embedded in the reducing citrate buffer ( $\mathrm{pH} \mathrm{6.0)}$ ), then the high-temperature treatment was carried on by heating in a water bath at $90-95^{\circ} \mathrm{C}$ for 30 minutes to unmask the sought antigens. After inhibition of endogenous peroxidase with 3\% solution of hydrogen peroxide on methanol the immunohistochemical reaction was performed by means of three-stage indirect enzyme-linked immunoassay using primary monoclonal antibodies (mAbs) to the following antigenic markers: neural-specific enolase (NSE), protein S-100 (S-100), vimentin (Vim), $\alpha$-smooth muscle actin ( $\alpha$-SMA) and epithelial membrane antigen (EMA). These primary monoclonal antibodies were used in dilutions according to the attached to them recommendations by company-manufacturer "Novocastra" (UK) - NSE (1:50), S-100 (1:50), Vim (1:100), $\alpha$-SMA (1:50), EMA (1:200). Results' visualization was performed by peroxidase activity detection by its histochemical revealing with the use of chromogenic substrate mixture based on 3-amino-9ethylcarbazole. The specificity of the sought antigen expression in test samples was confirmed by its lack in control sections, not treated with primary monoclonal antibodies. At the final stage the sections were stained again with hematoxylin and embedded in glycerolgelatin.

6). The mathematical results analysis of morphological, clinical and experimental study sections, followed by the statistical processing of the obtained information. The resulting digital data were processed statistically. Statistical significance of the results was determined with nonparametric Wilcoxon-Mann-Whitney test.

\section{Results and Discussion}

\subsection{General Clinical and Morphological Changes in the Capsule Cells and the Lens Substance in Different Types Formation of Age-Related Cataract}

During the ophthalmic diagnostics and retrospective analysis of outpatient cards' data of 198 patients of both sexes over 60 years the continuous statistical clinical study of patients successfully operated on for age-related cataract and applying to hospital within one year after surgery with secondary lens opacity signs was carried out. It was found that in all the examined persons from the group successfully operated on for the cortical type of age-related cataract (78 patients) in one year the proliferative type signs of secondary cataract (17 people) and secondary cataract of the fibrous type (one patient) were formed; whereas in the group successfully operated on for the nuclear type of age-related cataract (120 patients) secondary cataract of the fibrous type (14 patients) was detected (Table 1).

Table 1. Frequency of different types of age-related and secondary cataracts in humans.

\begin{tabular}{lll}
\hline Age-related cataract type & Age-related cortical cataract $(\mathbf{n}=\mathbf{7 8})$ & Age-related nuclear cataract $(\mathbf{n}=\mathbf{1 2 0})$ \\
\hline Proliferative type of secondary cataract & 17 patients $(22.1 \%)$ & Not revealed \\
Fibrous type of secondary cataract & 1 patient $(1.3 \%)$ & 14 patients $(11.6 \%)$ \\
\hline
\end{tabular}

In hematoxylin-eosin staining the sagittal paraffin sections of lens with cataract in their re-treatment in the postoperative period with secondary cataract signs we found the main morphological differences between types of age-related and secondary cataract.

In the proliferative type formation of secondary cataract in a patient, successfully operated on for the cortical type of age-related cataract, distinct hydration of the lens cortical part, dissociation of lens cells-fibers, sphenoidal spaces filled with detritus and vacuoles were determined, in addition, at the border of the cortical and nuclear parts the area of intense vacuolization was formed. The lens nuclear part was compressed with hydrated cortical masses, but it had no structural abnormalities. 
In the fibrous type development of secondary cataract in a patient, successfully operated on for the nuclear type of agerelated cataract, the sections' staining with hematoxylineosin revealed in the lens nucleus region the cluster of uniform, homogenous material, which increased it in volume, resulting in the unchanged cortical layer compression.

Clinical manifestations of cortical and nuclear types of age-related cataract did not have much similarity. Of interest was the large number of important differences, which included - the initial localization of opacity (in the lens cortical and nuclear parts), the presence or absence of the lens hydration signs (formation of water fissures, vacuoles, or the lens hardening with no hydration signs), different color of opacity (gray or brown, respectively). Thus clinical manifestations of proliferative and fibrous types of secondary cataract had no similarities either. They were united by a significant visual acuity reduction after successfully performed cataract surgery. The important differences between the proliferative and fibrous types of secondary cataract included - the reduction mechanism of the capsule transparency in the postoperative period (in the first case - it was the spherical cell conglomerates formation on the inner surface of the lens capsule from the remaining after surgical treatment of primary cataract lens epithelial cells, in the second - the posterior lens capsule fibrosis).

From the literature it is known that pathological morphology of the cortical type of age-related cataract is characterized by the appearance in the lens cortex water fissures, the cortex dissociation accumulating in the extracellular space with moisture. Then occurs opacity of the radial water fissures and formation of larger pin- and wedge-shaped opacities, which are gradually moving in the direction of the anterior and posterior lens capsule, and at the stage of mature cortical cataract the entire cortex is undergone to opacity, getting a grayish-white color ('gray' cataract). In rare cases, as a result of cataract hypermaturation due to lysis of muddy diluted cortical masses and the lens nucleus its spontaneous resorption can occur (Morgagni cataract). Such an outcome is characteristic only to the cortical type of age-related cataract [3, 4].

In the nuclear type formation of age-related cataract the initial opacities appear in the internal embryonic nucleus of the lens, and then spreading to all parts of the mature nucleus. Opacity has reddish or reddish-brown color ('brown' cataract), is homogeneous in nature and is located diffusely $[3,4]$. At the age-related nuclear cataract hypermaturation the excessive hardening of all lens substance is observed, and 'hard', 'brown' cataract is formed. The predominance of fibers sclerosis at their disintegration distinguishes the nuclear age-related cataract from the cortical cataract. The study of the ultrastructural disorders of the lens with nuclear cataract in elderly patients revealed the areas formation of its 'globular degeneration' [21], which consisted of spherical protein globules (multilamellar bodies, MLBs) with a diameter of 1 to 20 microns, coated with the lipid bilayer membrane capable of reducing the light transmittance of $65 \%$. The marked pathological changes of the lens were not found in patients with age-related cortical cataract [22].

Important pathogenetic differences in age-related cortical and nuclear cataract were repeatedly confirmed by experimental models cataractogenesis [16, 23].

It is important to note that in the postoperative period in patients with age-related cortical cataract the proliferative type of secondary cataract (with the formation of AdamyukElschnig pearls) is significantly more frequently formed, in patients with age-related nuclear cataracts - the fibrous type (the posterior lens capsule fibrosis) $[3,11]$.

Thus, the findings of important morphological and functional differences between the processes of age-related cortical and nuclear cataractogenesis suggest that exposure to the human lens of such causal factors as age, can be realized through completely different pathogenetic mechanisms, leading eventually to the certain types formation of complications, including postoperative (secondary cataract types).

\subsection{Neurotransmitter Bioamines Status of the Capsule Cells and the Lens Substances Normally and in Different Types of Age-Related Cataract}

In luminescent microscopy of intact lens sections treated by the Cross method, the lens nucleus was well visualized, its luminescence was expressed intensively, had an emeraldgreen color, and not faded for a long time. The luminescence of the intact lens nucleus was determined by the background luminescence of its central parts, which value according to spectrofluorometry on the average was $0.059 \pm 0.0014 \mathrm{mv}$ (Table 2). In the intact lens cortex area the luminescence was almost equal, but less expressed, it also had emerald-green color, but it was fading faster. The luminescence of intact lens cortex was determined by the background luminescence of lens fibers cytoplasm, forming its cortex, and on the average was $0.0511 \pm 0.0024 \mathrm{mv}$. It was found that the cells of the central part of the anterior lens capsule had less expressed luminescence $(0.0423 \pm 0.0018 \mathrm{mv})$ in comparison with the cells of its germinal zone $(0.0717 \pm 0.0023 \mathrm{mv})$.

Table 2. Comparative fluorescent-histochemical characterization of the lens substances and capsule (germinative zone) normally and at its age-related and secondary opacity in humans.

\begin{tabular}{|c|c|c|c|c|c|c|}
\hline \multirow{2}{*}{$\begin{array}{l}\text { Fluorescent-histochemical staining } \\
\text { method } \\
\text { Lens part }\end{array}$} & \multicolumn{3}{|c|}{ Method of Cross-Evan-Rost (level of histamine) } & \multicolumn{3}{|c|}{ Method of Falk-Hillarp (level of catecholamines) } \\
\hline & Cortex & Nucleus & $\begin{array}{l}\text { Lens capsule } \\
\text { (germinative zone) }\end{array}$ & Cortex & Nucleus & $\begin{array}{l}\text { Lens capsule } \\
\text { (germinative zone) }\end{array}$ \\
\hline Intact lens & $0.0511 \pm 0.0024$ & $0.059 \pm 0.0014$ & $0.0717 \pm 0.0023$ & $0.008 \pm 0.0005$ & $0.0077 \pm 0.0004$ & $0.0097 \pm 0.0007$ \\
\hline Age cortical and secondary proliferative cataract & $0.073 \pm 0.0029 * *$ & $0.0899 \pm 0.0028 * *$ & $0.0853 \pm 0.0025 * *$ & $0.0102 \pm 0.00025^{*}$ & $0.0121 \pm 0.0003 *$ & $0.0105 \pm 0.0007 *$ \\
\hline Age nuclear and secondary fibrous cataract & $0.0525 \pm 0.0041 *$ & $0.0637 \pm 0.0034 * *$ & $0.0613 \pm 0.0031 * *$ & $0.012 \pm 0.0002 *$ & $0.0147 \pm 0.0002 *$ & $0.012 \pm 0.0009 *$ \\
\hline
\end{tabular}


Table 2. Continued.

\begin{tabular}{llll}
\hline Fluorescent-histochemical staining method & Method of Falk-Hillarp (level of serotonin) & Nucleus & Lens capsule (germinative zone) \\
\hline Lens part & Cortex & $0.0238 \pm 0.0019$ & $0.0228 \pm 0.0014$ \\
Intact lens & $0.0175 \pm 0.0004$ & $0.0528 \pm 0.0012 *$ & $0.0285 \pm 0.0015^{*}$ \\
Age cortical and secondary proliferative cataract & $0.027 \pm 0.0015^{*}$ & $0.0833 \pm 0.0022^{*}$ & $0.0597 \pm 0.0024 *$ \\
Age nuclear and secondary fibrous cataract & $0.053 \pm 0.0015^{*}$ & \\
\hline
\end{tabular}

Comments: *- $\mathrm{P}<0.01 ; * *-\mathrm{P}<0.05$

At the age-related cortical cataract, complicated in the postoperative period with the proliferative type formation of secondary cataract, the histamine level was subject to significant changes. According to spectrofluorometry (Table 2 ), the histamine concentration in the lens cortex was increased by $42.9 \%$ (on the average $0.0730 \pm 0.0029 \mathrm{mv}$, $\mathrm{P}<0.05$ ). The luminescence intensity of the cortical and nuclear parts was greatly increased, got yellow-green shade, and was not fading for a long time. The luminescence in the lens nucleus, still remained the most intense on the section $(0.0899 \pm 0.0028 \mathrm{mv}, \mathrm{P}<0.05)$ and by $51.1 \%$ exceeded the histamine level in normal. The luminescence of the cells of the peripheral anterior lens capsule was $0.0853 \pm 0.0025 \mathrm{mv}$ $(\mathrm{P}<0.05)$, which was $18.9 \%$ higher than the histamine level in the intact capsule epithelial cells.

Another trend could be seen in the age-related nuclear cataract formation, the postoperative period was complicated by the fibrous type formation of secondary cataract. The luminescence of the lens sections was expressed moderately, it had emerald-green color, and visually the luminescence intensity in the cortex area and the lens nucleus was almost the same. In the lens cortex the histamine concentration was almost indistinguishable from that of the norm $(0.0525 \pm 0.0041 \mathrm{mv}, \mathrm{P}<0.01)$, the histamine level increased by only $2.7 \%$. In the lens nucleus area some $(7.1 \%)$ decrease in the histamine level (an average of $0.0637 \pm 0.0034 \mathrm{mv}, \mathrm{P}<0.05$ ) was found. The cells luminescence of the anterior lens capsule in the growth zone at the age-related nuclear cataract was $0.0613 \pm 0.0031 \mathrm{mv}(\mathrm{P}<0.05)$, which was $14.5 \%$ less than normal (Table 2).

The luminescence of intact lens stained by the Falk-Hillarp method to selective study the levels of catecholamines and serotonin in it, was expressed moderately, and had emeraldgreen color. Visually, the luminescence intensity of the lens nucleus prevailed to some extent over the luminescence in its cortex area. According to spectrofluorometry (Table 2), the catecholamine level in the cortical and nuclear parts of the intact lens was practically the same $(0.0080 \pm 0.0005 \mathrm{mv}$ and $0.0077 \pm 0.0004 \mathrm{mv}$, respectively). It was found that the serotonin concentration in the lens nucleus $(0.0238$ $\pm 0.0019 \mathrm{mv})$ prevailed over its level in the cortex $(0.0175 \pm 0.0004 \mathrm{mv})$. The cells of the central part of the anterior lens capsule stained by the Falk-Hillarp method had less expressed luminescence (catecholamines $0.0049 \pm 0.0004 \mathrm{mv}$, serotonin $0.0193 \pm 0.0012 \mathrm{mv}$ ) compared to its growth cells zone (catecholamine $0.0097 \pm 0.0007 \mathrm{mv}$, serotonin $0.0228 \pm 0.0014 \mathrm{mv}$ ).

In the cortical type formation of age-related cataract and the proliferative type of secondary cataract a significant increase in serotonin level (in the cortical lens section 1.5 times, in nuclear - 2.2 times) and a moderate increase in catecholamine concentration (in the lens cortex by $27,5 \%$, in the nucleus - by $57 \%$ ) were determined (Table 2 ). The level of catecholamines and serotonin in the cells of the peripheral anterior lens capsule with cortical cataract was $0.0105 \pm 0.0007 \mathrm{mv}(\mathrm{P}<0.01)$ and $0.0285 \pm 0.0015 \mathrm{mv}(\mathrm{P}<0.01)$, that, respectively, 8.3 and $25 \%$ higher than the histamine level in the intact capsule cells.

The development of the nuclear type of age-related and the fibrous type of secondary cataract was accompanied by a sharp (3.5 times) increase of serotonin concentration in the lens nucleus, the serotonin level in the cortical part was also significantly increased (3 times). The catecholamines concentration in the lens nucleus was increased to a greater extent $(90.9 \%)$ than in the area of its cortex $(50 \%)$. The level of catecholamines and serotonin in the cells of the peripheral anterior lens capsule with nuclear cataract was $0.0120 \pm 0.0009 \mathrm{mv}(\mathrm{P}<0.01)$ and $0.0597 \pm 0.0024 \mathrm{mv}(\mathrm{P}<0.01)$, that, respectively, 1.2 and 2.6 times more than normal (Table 2).

When analyzing the obtained results, we judge from the fact that modern information, characterizing the biogenic amines role in the lens metabolic processes, evidences both participation in the course of the pathological process [16], and the importance of their properties in the process of division, growth and differentiation of cells [17].

According to Williams M. R. et al. (2001) [17] the agonists (histamine, carbachol, sulfur) and the calcium entry antagonists are stored in the lens intracellularly. Their system provides the signaling mechanism for preventing calcium overload of the lens. Detected in the present study the fact of a significant increase in the histamine level in the formation of age-related cortical cataract and secondary proliferative cataract suggests that the failure of this signaling mechanism is essential for the formation of precisely the cortical type of cataract and secondary cataract with Elshnig pearls formation, which is evidenced by scientific literature data on the experimental model of cortical cataract recreating by incubating the intact lens in the calcium-rich environment [16].

The manifestations associated with implementation of physiological [24] and pathological [25] effects of catecholamines in the eye tissues are also important and multifaceted. It is known that the epinephrine intravitreal injection into rabbit leads to the development of experimental cortical cataract with swelling, stratification and disintegration of the lens fibers-cells.

It was proved that serotonin had a stimulating effect on the 
processes of growth and differentiation of the eye cells, triggered important pathological effects - the retinal amacrine cells change, the increased intraocular pressure, the myosis and vasoconstriction of the eye [26].

Comparing bioamines supply of different types formation processes of age-related and secondary cataract, a pattern was found. In formation of the cortical type of age-related cataract and proliferative type of secondary cataract a significant increase in the concentration of histamine, serotonin and catecholamines in the epithelium, cortex and lens nucleus was revealed. For formation of the nuclear type of age-related and fibrous type of secondary cataract a significant increase in the serotonin level, and a moderate increase of the catecholamine concentration mainly in the nucleus of the lens, while the histamine level is virtually identical to the intact lens indices, was characteristic.

Thus, this study revealed significant differences in the bioamines supply of the epithelium and the lens material in the formation of postoperative proliferative and fibrous types of secondary cataract in humans.

\subsection{Phenotype of the Capsule Cells and the Lens Material Normally and in Different Types of Age-Related Cataract}

When conducting immunohistochemical reactions with monoclonal antibodies to neural-specific enolase (NSE), protein S-100 (S-100), vimentin (Vim), epithelial membrane antigen (EMA), $\alpha$-smooth muscle actin ( $\alpha$-SMA) in all parts of the intact human lens the specific staining was not detected.

In the immunohistochemical staining of the epithelium and the substance of the human lens with the cortical and nuclear types of age-related cataract a number of important differences was revealed.

In the age-related cortical cataract, complicated in the postoperative period by the proliferative type formation of secondary cataract, the homogeneous, immuno-positive reaction for antibodies to S-100 protein, Vim and NSE was established, more expressed for antibodies to NSE. By immunohistochemistry in the cell cytoplasm of the equatorial (germinal) epithelium of the lens area, affected with agerelated cortical cataract, the intense accumulation of immunopositive granules to the NSE, S-100 protein and Vim was detected. In the wedge-shaped spaces between the swollen cells-fibers of the lens cortex, filled with detritus and vacuoles, a large concentration of the immunospecific reaction product to the NSE, S-100 protein and Vim was also found. The moderately expressed immunopositive reaction to the NSE, S100 protein and Vim was detected in vacuolization zone, located on the border of the cortical and nuclear parts of the lens material. It is important to note, that in the lens nuclear part the immunoreactive mark to the named antibodies was not accumulated. In addition, the lens sections, affected with agerelated cortical cataracts the immunopositive reaction to $\alpha$ SMA and EMA were not detected.

Another trend could be seen in age-related nuclear cataract, the postoperative period of which was complicated by the fibrous type formation of secondary cataract. By immunohistochemistry in the lens nucleus the immunopositive reaction to monoclonal antibodies to $\alpha$-SMA and EMA was detected, which had a distinct local character. It was revealed that lens fibers-cells, forming its cortical layer no specific staining for $\alpha$-SMA and EMA was shown in any case. But anterior epithelial lens cells had specific staining for EMA and $\alpha$-SMA. When using monoclonal antibodies to NSE, S-100 protein and Vim lens sections in the age-related cataract nuclear formation of no specific staining was shown in any case.

In the age-related cortical cataract development in the human lens the expressed immunopositive reaction for specific monoclonal antibodies (NSE, S-100 protein and Vim) was detected, wherein the occurrence region of the specific reaction was outlined with the cortical part of the lens substance and its epithelium.

In the age-related nuclear cataract formation the specific staining was observed only in the lens nucleus, and the occurrence of immunopositive reactions was due to the use of monoclonal antibodies to $\alpha$-SMA and EMA.

Modern experimental medicine has information confirming the importance of preserving the tissue phenotypic characteristics for its functions immutability. On the human lens material in cataract different types formation the positive reaction to vimentin was revealed and the assumption about the lens epithelial cells transformation in cataract into mesenchymal ones was put forward [7]. In the lens cell culture it was shown that the disturbing factors effects initiated their transformation into myofibroblasts [6]. These studies involve a fundamental problem of cell phenotype transformation in different conditions and show the lens cells changes in phenotypic characteristics in its pathology.

Analysis of the present study data leads to the conclusion that the specific patterns detection of the lens cells phenotype transformation, occurring in its pathological aging in humans. For the formation of the cortical type of age-related cataract and proliferative type of secondary cataract the mesenchymal-neuroectodermal phenotype transformation direction of the lens cells is typical. It was found that the development of age-related nuclear cataracts and secondary fibrous cataract was accompanied by myoepithelial direction formation. This is consistent with the researchers who claimed the existence of phenomena influenced by the cell phenotype transformation of a number of external and internal factors $[6,7,27,28]$. The epithelial phenotype transition into the mesenchymal cells, called epithelialmesenchymal transition, which is recognized as a fundamental process, controlling morphogenesis in multicellular organism, is most mentioned [29, 30, 31].

Analyzing the results of the immunohistochemical part of the study, it should be emphasized that the normal human lens cells do not show immunoreactivity to the NSE, protein S-100, Vim, $\alpha$-SMA and EMA, therefore, in the intact lens there are no immunohistochemical marks on the basic tissues types. The reason for this is probably the intactness of the 
blood-barrier, providing the structure of the eyeball with 'overbarrier' status. However, it was proved that in the occurrence of age-related diseases of the eye, including cataracts, an important role belonged to the nervous trophic regulation disturbance, followed by a change in the bloodbarrier penetration and phenotypic characteristics of the affected organ cells $[3,6,9,11,32]$.

Thus, the results of immunohistochemical studies revealed the significant flexibility of the epithelial cells phenotype and the lens substances in a human in normal, at its age-related and secondary opacity, indicating that the certain type formation of age-related and secondary cataract depended on the lens cells change of its phenotypic characteristics.

Study findings:

1. It was found that in patients over 60 years a significant levels increase histamine (up to $0.073 \pm 0,0029 \mathrm{mv}$, $\mathrm{P}<0.05$ ), catecholamines (up to $0.0102 \pm 0.00025 \mathrm{mv}$, $\mathrm{P}<0.01$ ) and serotonin (up to $0.027 \pm 0.0015 \mathrm{mv}, \mathrm{P}<0.01$ ) in the lens promoted the proliferative type formation of secondary cataract, whereas the content increase of serotonin (up to $0.0833 \pm 0.0022 \mathrm{mv}, \quad \mathrm{P}<0.01$ ) and catecholamines (up to $0.0147 \pm 0.0002 \mathrm{mv}, \mathrm{P}<0.01$ ) - the fibrous type.

2. The expressed human lens cells immunopositivity on monoclonal antibodies to NSE, S-100 protein and Vim, promoting to the proliferative type formation of secondary cataract, was revealed, whereas the specified immunopositivity on monoclonal antibodies to $\alpha$-SMA, EMA - the fibrous type.

3. It was noted that in the postoperative period in patients with age-related cortical cataract the proliferative type of secondary cataract was significantly more frequently formed, in patients with age-related nuclear cataract the fibrous type.

\section{Conclusion}

The revealed changes complex in the lens in its pathological aging includes the high flexibility of neurotransmitter bioamines supply and immunohistochemical state of the epithelium and human lens substance even after the successful surgical treatment of agerelated cataract its post-operative complications formation secondary cataract of the proliferative or fibrous type.

The revealed similarities in changing of neurotransmitter status, immunoreactivity of the epithelium and human lens fibers in different types of age-related cataract (cortical and nuclear) and at its postoperative complications (proliferative and fibrous type of secondary cataract, respectively), can serve as the important evidence of their common pathogenesis, and therefore, the type of age-related cataract is to be proposed as a marker available for high risk predicting of postoperative secondary cataract of certain types and carrying out its early, differentiated prevention.

The results allow for the first time to put forward a new concept of the secondary cataract pathogenesis: the nervous trophism disturbance, determining the age-related neuro- degenerative process direction of organs and tissues, causes the lens cells, remaining after surgical excision, to pathologic regeneration in previously formed direction.

The presented concept of the secondary cataract pathogenesis stresses the importance of the fundamental problems of medicine - the tissue differentiation stability maintaining of a living organism in a variety of physiological and pathological conditions.

\section{Acknowledgements}

This work was performed with financial support of Ministry of education and science of Russian Federation within program 'Scientific and Scientific-Pedagogical People of Innovative Russia' (agreement with the researcher No. 14. B37.21.0221).

\section{References}

[1] C. M. Hernandez (2010). Cataracts: Causes, Symptoms, and Surgery. New York, USA, ISBN 978-1-61668-955-1.

[2] G. L. Kanthan et al. (2008). Ten-Year Incidence of AgeRelated Cataract and Cataract Surgery in an Older Australian Population. The Blue Mountains Eye Study. Ophthalmology, 115:808-814.

[3] E. V. Malcev and K. P. Pavluchenko (2002). Biological Features and Diseases of Lens. Odessa, Ukraine. ISBN 966549-716-2.

[4] N. V. Korsakova (2012). Modern Data about Age-Related Cataract Pathogenesis in Humans. New York, USA. ISBN 978-1-62081-823-7.

[5] N. V. Korsakova et al. (2012). Morphological Basis of Desympathization of the Eye as a New Means of Experimental Modeling of Cataract. Neuroscience and Behavioral Physiology, 42:1024-1028.

[6] S. Saika et al. (2013). Response of Lens Epithelial Cells to Injury: Role of Lumican in Epithelial Mesenchymal Transition. Invest Ophthalmol Vis Sci, 44:2094-2102.

[7] A. Synder et al. (2002). A Study of Human Lens Epithelial Cells by Light and Electron Microscopy and by Immunohistochemistry in Different Types of Cataracts. Klin Oczna, 104:369-373.

[8] N. V. Korsakova et al. (2008). Immunohistochemical Analysis of Lens Cells on Formation of Different Types of Age-Related Cataract in Humans. Neuroscience and Behavioral Physiology, 38: 887-890.

[9] N. V. Korsakova et al. (2010). Discussing the Problem of Age-Related Cataract Pathogenesis in Human: Morphological and Immunohistochemical Aspects. In: C. M. Hernandez. Cataracts: Causes, Symptoms, and Surgery. NovaPublishers, New York, USA, pp 159-173.

[10] O. Sveinsson (1993). The Ultrastructure of Elschnig's Pearls in a Pseudophakic Eye. Acta Ophthalmol (Copenh), 71:95-98.

[11] N. V. Korsakova et al. (2013). Immune Reactivity of Human Lens Structures in Norm, Age-Related Cortical and Secondary Opacification. Morfologiia, 143:28-31. 
[12] G. Duncan and D. J. Collison (2003). Role of the NonNeuronal Cholinergic System in the Eye: a Review. Life Sci, 72:2009-2013.

[13] P. D. Gurta et al. (2005). Sex Hormone Receptors in the Human Eye. Surv Ophthalmol, 50:274-284.

[14] L. Wang et al. (2005). Sigma Receptor Antagonists Inhibit Human Lens Cell Growth and Induce Pigmentation. Invest Ophthalmol Vis Sci, 46:1403-1408.

[15] L. Wang et al. (2005). Growth Factor Receptor Signaling in Human Lens Cells: Role of the Calcium Store. Exp Eye Res, $80: 885-895$.

[16] L. Lorand et al. (1985). Formation of a 55 000-Weight CrossLinked Beta Crystallin Dimer in the $\mathrm{Ca}^{2+-}$ Treated Lens. A Model for Cataract. Biochemistry, 24:1525-1531.

[17] M. R. Williams et al. (2001). Role of the Endoplasmic Reticulum in Shaping Calcium Dynamics in Human Lens Cells. Invest Ophthalmol Vis Sci, 42:1009-1017.

[18] S. A. Cross et al. (1971). A Study of Methods Available for Cyto-Chemical Localization of Histamine by Fluorescence Induced with o-Phtaldehyde or Acetaldehyde. Histochem J, 3:471-476.

[19] B. Falk (1965). Observations on the Possibilities of the Cellular Localization of Monoamines by a Fluorescence Method. Acta Physiol Scand, 56:197-201.

[20] B. Falk et al. (1962). Fluorescence of Catecholamines and Related Compounds Condensed with Formaldehyde. J Histochem Cytochem, 10:348-354.

[21] K. O. Gilliland et al. (2001). Multilamellar Bodies as Potential Scattering Particles in Human Age-Related Nuclear Cataracts. Mol Vis, 22:120-130.

[22] C. D. Freel et al. (2002). Fourier Analysis of Cytoplasmic Texture in Nuclear Fiber Cells from Transparent and Cataractous Human and Animal Lenses. Exp Eye Res, 74:689-702.
[23] C. D. Freel et al. (2003). Ultrastructural Characterization and Fourier Analysis of Fiber Cell Cytoplasm in the Hyperbaric Oxygen Treated Guinea Pig Lens Opacification Model. Exp Eye Res, 76:405-415.

[24] G. Marchini et al. (2003). Comparative Study of the Effects of $2 \%$ Ibopamine, $10 \%$ Phenylephrine, and $1 \%$ Tropicamide on the Anterior Segment. Invest Ophthalmol Vis Sci, 44:281-289.

[25] I. Giuffre et al. (2004). The Effects of 2\% Ibopamine Eye Drops on the Intraocular Pressure and Pupil Motility of Patients with Open-Angle Glaucoma. Eur J Ophthalmol, 14:508-513.

[26] A. George et al. (2005). Retinal Serotonin, Eye Growth and Myopia Development in Chick. Exp Eye Res, 81:616-625.

[27] J. Bariety et al. (2003). Glomerular Epithelial-Mesenchymal Transdifferentiation in Pauci-Immune Crescentic Glomerulonephritis. Nephrol Dial Transplant, 18:1777-1784.

[28] A. Y. Demir et al. (2004). Menstrual Effluent Induces Epithelial-Mesenchymal Transitions in Mesothelial Cells. Hum Reprod, 19:21-29.

[29] J. P. Thiery (2003). Epithelial-Mesenchymal Transitions in Development and Pathologies. Curr Opin Cell Biol, 15:740746.

[30] X. Wu et al. (2016). Bit1-a Potential Positive Regulator of Epithelial-Mesenchymal Transition in Lens Epithelial Cells. Graefes Arch Clin Exp Ophthalmol, 254(7):1311-1318.

[31] Y. K. Zhonghua et al. (2016). TAK1 Promotes EpithelialMesenchymal Transition of Lens Epithelial Cells. Chin J Ophthalmol, 52:278-284.

[32] C. Zhang et al. (2016). SiRNA Targeting mTOR Effectively Prevents the Proliferation and Migration of Human Lens Epithelial Cells. PLoS One, 11(12): e0167349. 